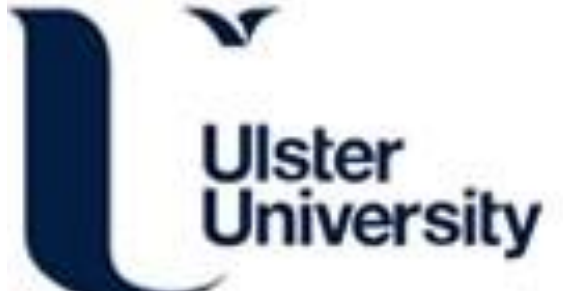

\section{Interpolating Low Amplitude ECG Signals Combined with Filtering According to International Standards Improves Inverse Reconstruction of Cardiac Electrical Activity}

Rababah, A., Finlay, D., Bear, L., Bond, RR., Rjoob, K., \& McLaughlin, J. (2019). Interpolating Low Amplitude ECG Signals Combined with Filtering According to International Standards Improves Inverse Reconstruction of Cardiac Electrical Activity. Lecture Notes in Computer Science, 11504, 112-120. https://doi.org/10.1007/978-3030-21949-9_13

Link to publication record in Ulster University Research Portal

\section{Published in:}

Lecture Notes in Computer Science

Publication Status:

Published (in print/issue): 30/05/2019

DOI:

10.1007/978-3-030-21949-9_13

\section{Document Version}

Author Accepted version

\section{General rights}

Copyright for the publications made accessible via Ulster University's Research Portal is retained by the author(s) and / or other copyright owners and it is a condition of accessing these publications that users recognise and abide by the legal requirements associated with these rights.

\section{Take down policy}

The Research Portal is Ulster University's institutional repository that provides access to Ulster's research outputs. Every effort has been made to ensure that content in the Research Portal does not infringe any person's rights, or applicable UK laws. If you discover content in the Research Portal that you believe breaches copyright or violates any law, please contact pure-support@ulster.ac.uk. 


\title{
Interpolating low amplitude ECG Signals Combined with Filtering According to International Standards Improves Inverse Reconstruction of Cardiac Electrical Activity
}

\author{
Ali Rababah ${ }^{1}$, Dewar Finlay ${ }^{1}$, Laura Bear ${ }^{2,3,4}$, Raymond Bond ${ }^{1}$, Khaled Rjoob ${ }^{1}$, and \\ James Mclaughlin ${ }^{1}$ \\ ${ }^{1}$ Faculty of Computing, Engineering and the Built Environment, Ulster University, Shore \\ Road, Newtownabbey, United Kingdom \\ Rababah-AQulster.ac.uk \\ ${ }^{2}$ IHU Liryc, Electrophysiology and Heart Modeling Institute, Fondation Bordeaux Universi- \\ té, F-33600 Pessac- Bordeaux, France \\ ${ }^{3}$ Univ. Bordeaux, Centre de recherche Cardio-Thoracique de Bordeaux, U1045, F-33000, \\ Bordeaux, France \\ ${ }^{4}$ INSERM, Centre de recherche Cardio-Thoracique de Bordeaux, U1045, F-33000 Bor- \\ deaux, France
}

\begin{abstract}
In this paper, the effect of reducing noise from ECG signals is investigated by applying filters in compliance with the standards for ECG devices, removing and interpolating low amplitude signals. Torso-tank experiment data was used with electrical activity recorded simultaneously from 128 tank electrodes and 108 epicardial sock electrodes. Subsequently, 10 representative beats were selected for analysis. Tikhonov zero-order regularization method was used to solve the inverse problem for the following groups; raw fullset, filtered fullset, raw low amplitude removed, filtered low amplitude removed, raw low amplitude interpolated, filtered low amplitude interpolated torso signals. Pearson's correlation was used for comparison between measured and computed electrograms and between activation maps derived from them. Filtering the signal according to the standards improved the reconstructed electrograms. In addition, removal of low amplitude signals and replacing them with interpolated signals combined with filtering according to the standard significantly improved the reconstructed electrograms and derived activation maps.
\end{abstract}

\section{Introduction}

Electrocardiographic imaging (ECGI) is a reconstruction of cardiac electrical activity from high number of body surface electrodes and a patient specific heart-torso geometry. It is noticeable that ECGI has attracted the attention of both industry and academic researchers in the recent decade. It is a promising technique for guiding ablation therapy for atrial and ventricular arrhythmias[1]. The error in non-invasive electrocardiographic imaging (ECGI) reconstruction can be partially attributed to two main sources 
of signal noise. Firstly, low amplitude ECG signal recorded from the tank electrodes located away from the heart. Secondly, noises that contaminate ECG signal such as baseline drift, muscle artifact, and power line interference. In this paper, we will investigate the effect of removal or interpolating these low amplitude ECG signals on the inverse reconstruction of cardiac electrical activity which has not been investigated in the literature yet. In addition, we investigate the impact of applying a designed digital filter that complies with International and American standards for diagnostic ECG devices on the inverse reconstruction of epicardial potentials. We evaluate both filtering and interpolation based on a data recorded from both heart and tank surfaces in torso-tank experiment that contain an explanted pig's heart [2].

\section{Methods}

\subsection{Torso-Tank Experimental Setup}

All experimental data was obtained in accordance with the guidelines from Directive 2010/63/EU of the European Parliament on the protection of animals used for scientific purposes and approved by the local ethical committee and has previously been described in [2]. An explanted pig's heart was suspended in an instrumented, human shaped, electrolytic torso tank. Electrical potentials were recorded simultaneously from 128 tank electrodes and 108 sock electrodes at $2 \mathrm{kHz}$ (BioSemi, the Netherlands) and referenced to a Wilson's central terminal. Following that, 3D rotational fluoroscopy (Artis, Siemens) was used to acquire heart and tank geometries and locations of electrodes. 

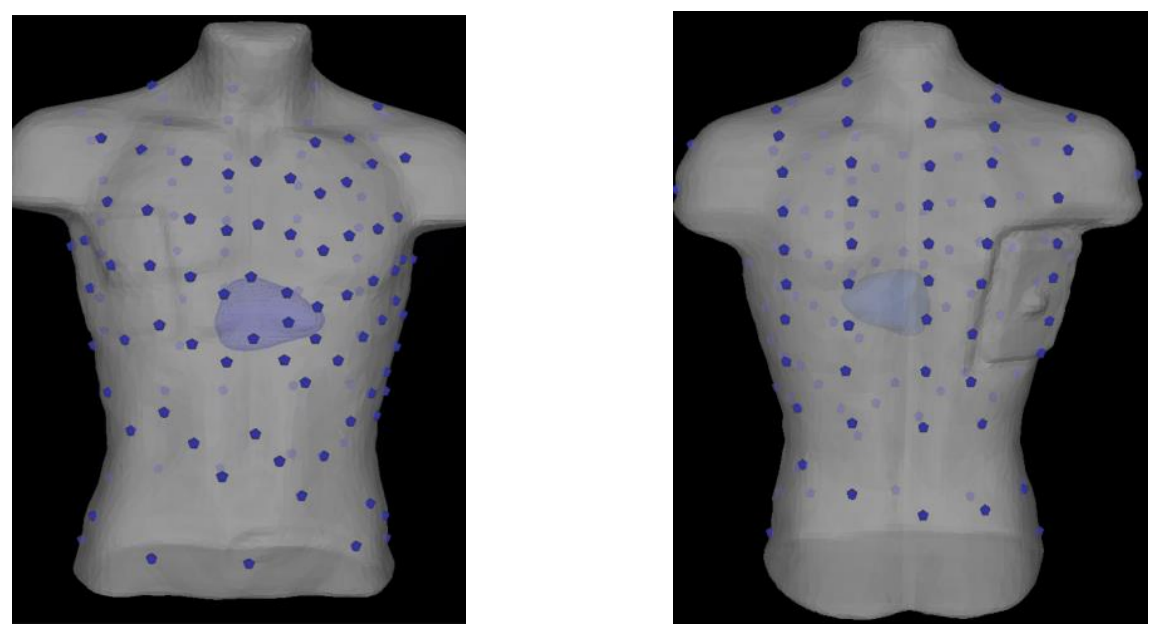

Fig. 1. The geometry of the heart and torso tank with the locations of tank electrodes marked in blue a) anterior view. b) posterior view [3].

\subsection{Removal of Low Amplitude ECG Signals}

After manual investigating of ECGs recorded from all tank electrodes, it appeared that the signals recorded from the inferior anterior and inferior posterior region (Fig. 1) of the tank have low amplitude $(<0.2 \mathrm{mV}$ peak to peak). To investigate the impact of these low amplitude signals (21 tank signals) on ECGI reconstruction, the inverse problem was solved before and after discarding these signals and solutions compared.

\subsection{Interpolating Low Amplitude ECG Signals}

Laplacian interpolation [4][5] has been reported in the literature for interpolating missing information in body surface potential maps. In this paper, 21 low amplitude ECG signals $(<0.2 \mathrm{mV}$ peak to peak) were removed, and the remaining 107 tank signals were used to interpolate signals at these locations. Epicardial potentials were calculated by solving the inverse problem before and after the interpolation and solution compared. 


\subsection{ECG Signal Filtering}

High-Pass Filter for Baseline Wander Removal. The change of electrical impedance at the skin-electrode interface which is resulted from subject movement, respiration, and perspiration appears in the recorded ECG signal as a low frequency noise called baseline wander (Fig. $2 \mathrm{a} \& 2 \mathrm{~b}$ ). This noise may hinder important ECG features such as ST-segment which is an important indicator for the diagnosis of myocardial infarction and ischemia. Hence, the suppression of baseline wander while preserving the ST-segment and other ECG components is the desired goal.

Current international IEC 60601-2-51, and American Standard AAMI EC11 for diagnostic electrocardiographic devices adopt the following recommendation[6]:

- cut-off frequency $(-3 \mathrm{~dB})$ of the high pass filter shall not exceed $0.5 \mathrm{~Hz}$.

- applying a $0.3 \mathrm{mV}$-s test signal to the high pass filter shall not result in a displacement in the baseline of more than $0.1 \mathrm{mV}$.

- passband ripple within $0.67-40 \mathrm{~Hz}$ range shall not exceed $0.9 \mathrm{~dB}$ [6].

There are plenty of available digital filters to pick from. Infinite Impulse Response (IIR) Butterworth Filter is the right selection when it comes to the flatness in the passband. A high pass Butterworth filter with a cut-off frequency $(-3 \mathrm{~dB})$ of $0.5 \mathrm{~Hz}$ and order 3 was designed and implemented in the forward and backward direction to ensure the compliance with the previously mentioned standards.

Power Line Frequency Removal. The contamination of ECG signal by $50 / 60 \mathrm{~Hz}$ power line interference is a major problem that prevents accurate interpretation and may lead to a misdiagnosis (Fig. 2d). Digital filters are used to eliminate this noise or reduce its impact. However, inaccurate implementation of these filters can cause an unacceptable ringing effect in the ECG signal after the QRS complex. This is caused when using a filter with high order. AAMI standards does not have an item for testing the ringing effect of a notch filter [7]. However, the British and European standard BS EN 60601-2-51:2003 states that the ringing peak to peak noise should not exceed 50 $\mu \mathrm{V}$ when a test ECG signal ANE20000 is used to test the filter. Since this signal is a closed source and it is difficult to access, we have used a simulated triangular signal of $3 \mathrm{mV}$ amplitude and $100 \mathrm{~ms}$ duration to test the ringing effect of our notch filter. The resulting peak to peak ringing noise is $24 \mu \mathrm{V}$ which is below $50 \mu \mathrm{V}$ requested by the European and British standard [7]. 

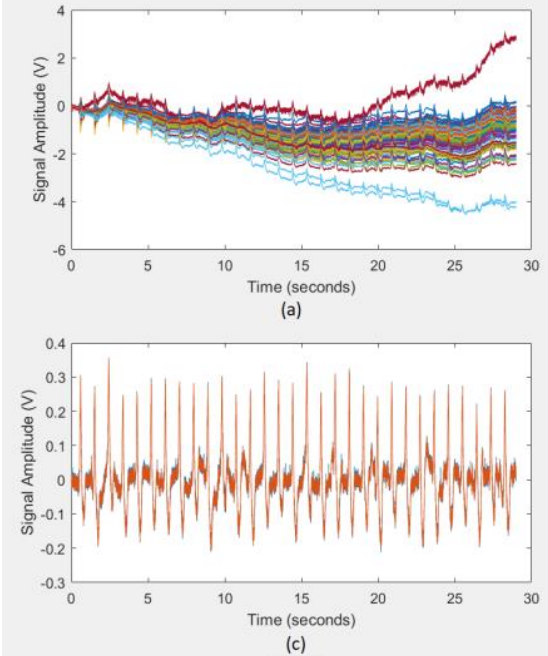
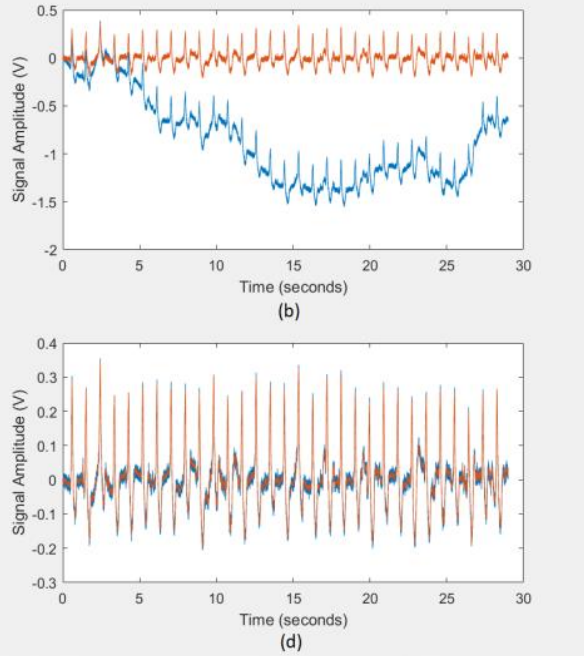

Fig. 2. a) Raw signal recorded from 128 torso electrodes. b) ECG signal at electrode number 1 before (blue) and after (red) baseline wander $(0.5 \mathrm{~Hz}$, order 3, 0-phase). c) ECG signal at electrode number 1 before (blue) and after (red) removing High frequency noise $(150 \mathrm{~Hz}$, order 3 , 0-phase). d) ECG signal at electrode number 1 before (blue) and after (red) removing $50 \mathrm{~Hz}$ line interference.

Low-Pass Filter for the Suppression of High Frequency Interference. High frequency noises such as muscle artifacts corrupt ECG signals and may hide some important features needed for ECG interpretations (Fig. 2c). The recommendation of American Heart Association (AHA) for diagnostic electrocardiographic devices is to use low-pass filters with cut-off frequency at $150 \mathrm{~Hz}$ to prevent the distortion of the QRS complex and maintain the small high frequency notches needed for diagnosis [8]. We have used a Butterworth low-pass filter with cut-off frequency at $150 \mathrm{~Hz}$ and order 3 to eliminate the high frequency noise from the signal.

\subsection{Inverse Reconstruction of Epicardial Potentials}

To solve the inverse problem, a forward transformation matrix that relates the epicardial potentials (894 nodes) to torso tank surface potentials (107 or 128 nodes) was calculated using the boundary element method (BEM) assuming the conductivity of the volume between heart surface and tank surface is homogenous [9]. We solved the inverse problem using Tikhonov zero-order regularization method from a toolkit for forward/inverse problems in electrocardiography within the SCIRun environment [3].

Ten beats were selected for the analysis (Fig 3). These signals were processed in different ways; filtering, removal of low amplitude signals, and interpolating low amplitude signals. The resulting signals; raw fullset signals, filtered fullset signals, raw (low amplitude removed) signals, filtered (low amplitude removed) signals, raw (low amplitude interpolated) signals, filtered (low amplitude interpolated) signals, were 
used for solving the inverse problem leading to 6 different solutions for each beat (Fig 4). Comparison was performed between the computed electrograms and the measured electrograms (Ground Truth) using Pearson's correlation coefficient. Activation times were defined by fitting a global activation field to activation delays between electrograms [10]. Activation was also compared using Pearson's correlation. Student's t-test was used for statistical analysis with statistical significance defined for $\mathrm{p}<0.05$.

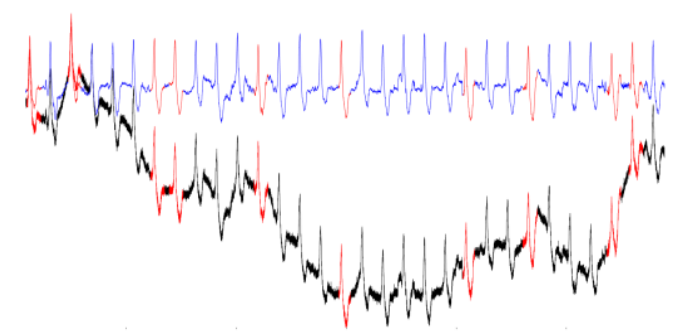

Fig. 3. Raw vs filtered ECG signal recorded at one of the electrodes. Ten representative beats marked in red were selected for analysis.

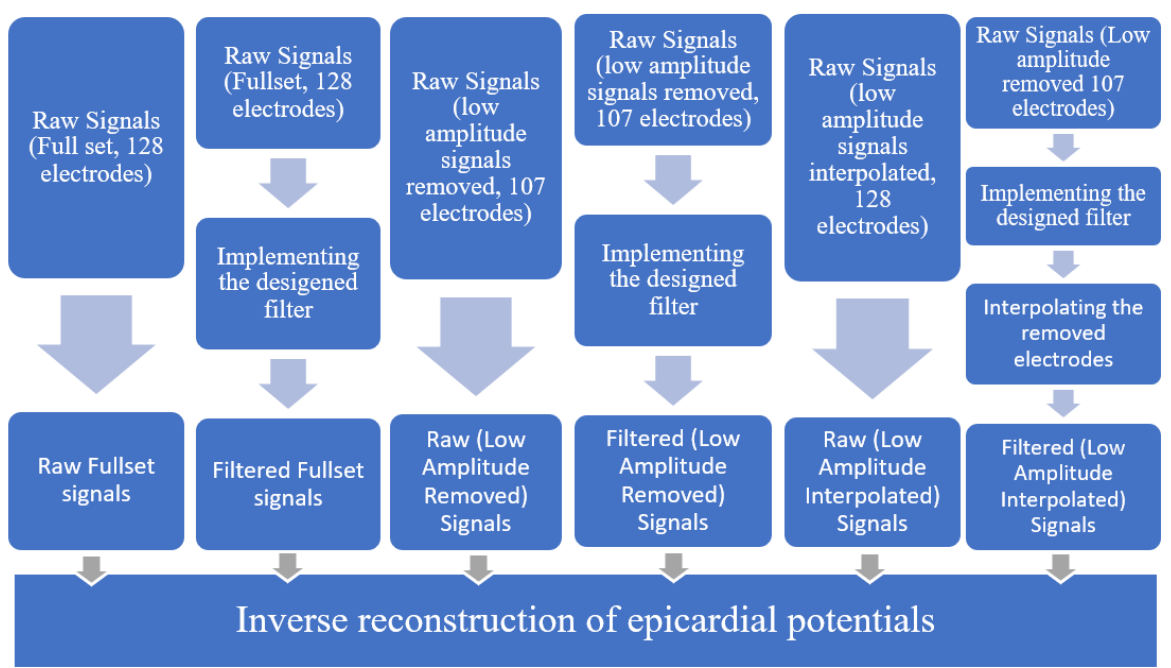

Fig 4. Flow diagram which shows the protocol followed to prepare tank signals for the inverse reconstruction.

\section{Results}

The median correlation between computed and measured EGMs of the 10 beats are as shown in Fig. 5. For the three categories of signals (fullset, low amplitude removed, low amplitude interpolated), filtering the signal according to the standards significant- 
ly improved the inverse reconstruction of epicardial potentials $(p<0.01)$. Comparing between filtered signals showed that epicardial reconstruction better for filtered LAinterpolated signals than for filtered fullset signals which was better than that for filtered LA-removed signals $(\mathrm{p}<0.05)$.

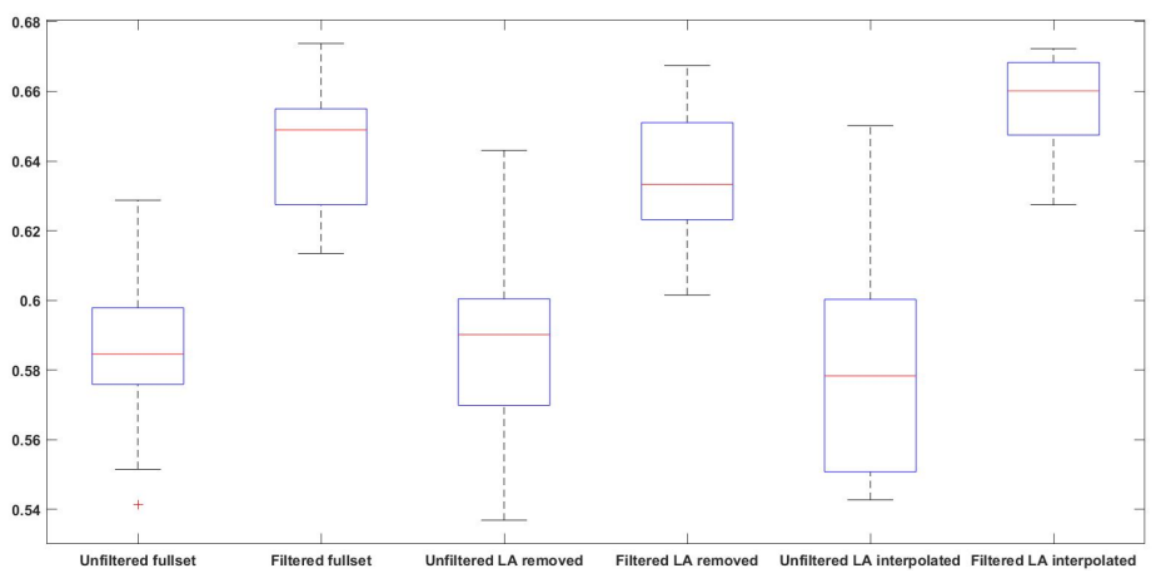

Fig. 5. Comparison of median correlation between different groups. Filtered fullset $>$ unfiltered fullset $(p<0.01)$, Filtered LA removed $>$ unfiltered LA removed $(p<0.01)$, Filtered LA interpolated $>$ unfiltered LA interpolated $(\mathrm{p}<0.01)$, Filtered LA interpolated $>$ Filtered fullset $>$ Filtered LA removed $(\mathrm{p}<0.05)$.

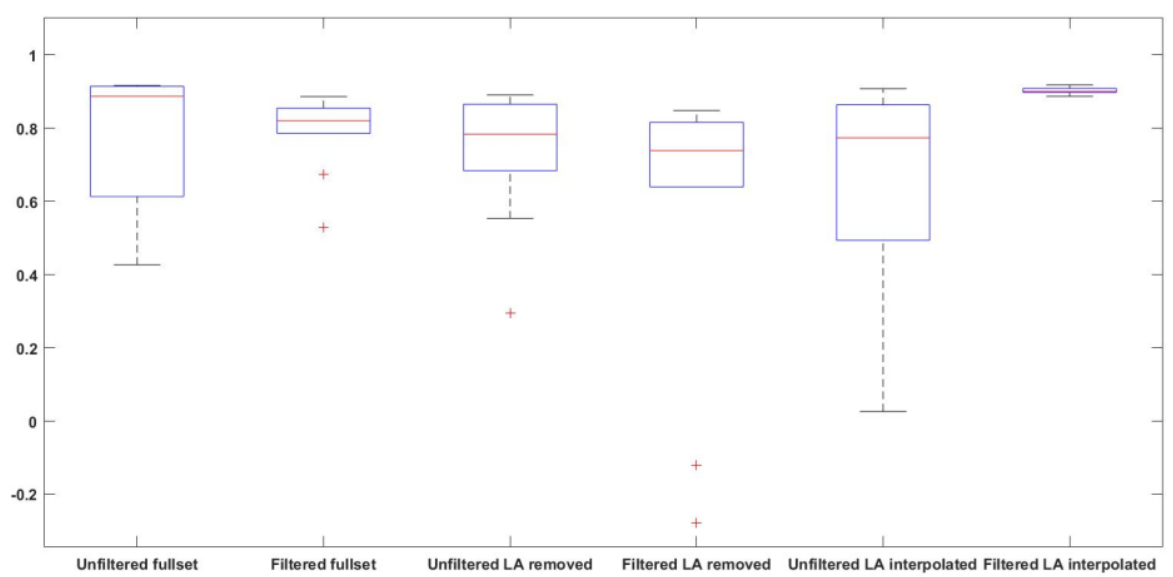

Fig. 6. Correlation between computed activation times and measured activation times for different groups. Filtered LA interpolated $>$ all other groups $(\mathrm{p}<0.05)$. difference between any other groups is insignificant $(\mathrm{p}>0.1)$

Fig. 6 shows that when comparing between computed and measured activation times, the filtered LA-interpolated signals resulted in the best solution as compared to 
any other filtered or unfiltered signals $(p<0.05)$. there is no significant different between any other groups shown in Fig. $6(\mathrm{p}>0.1)$. Fig. 7 shows the high correlation between Activation maps derived from recorded and reconstructed electrograms using filtered LA-interpolated torso signals for the seventh selected beat.

\section{Discussion}

Filtering tank ECG signals is an important processing method for a better inverse reconstruction of epicardial potentials. However, careful filtering (zero-phase or linear phase response, $0.5-150 \mathrm{~Hz}$ notched at $50 \mathrm{~Hz}$, lower than $50 \mathrm{uV}$ peak to peak ringing artifact) that is according with international and American standard for ECG diagnostic devices[6] should be implemented to guarantee this improvement in the reconstruction. Low amplitude ECG signals $(<0.2 \mathrm{mV})$ is another source of error that impacts the inverse solution. Removal of these signals and replacing them with interpolated signals significantly improved the reconstructed EGMs and activation times as shown in Fig. 5, Fig. 6 and Fig. 7.

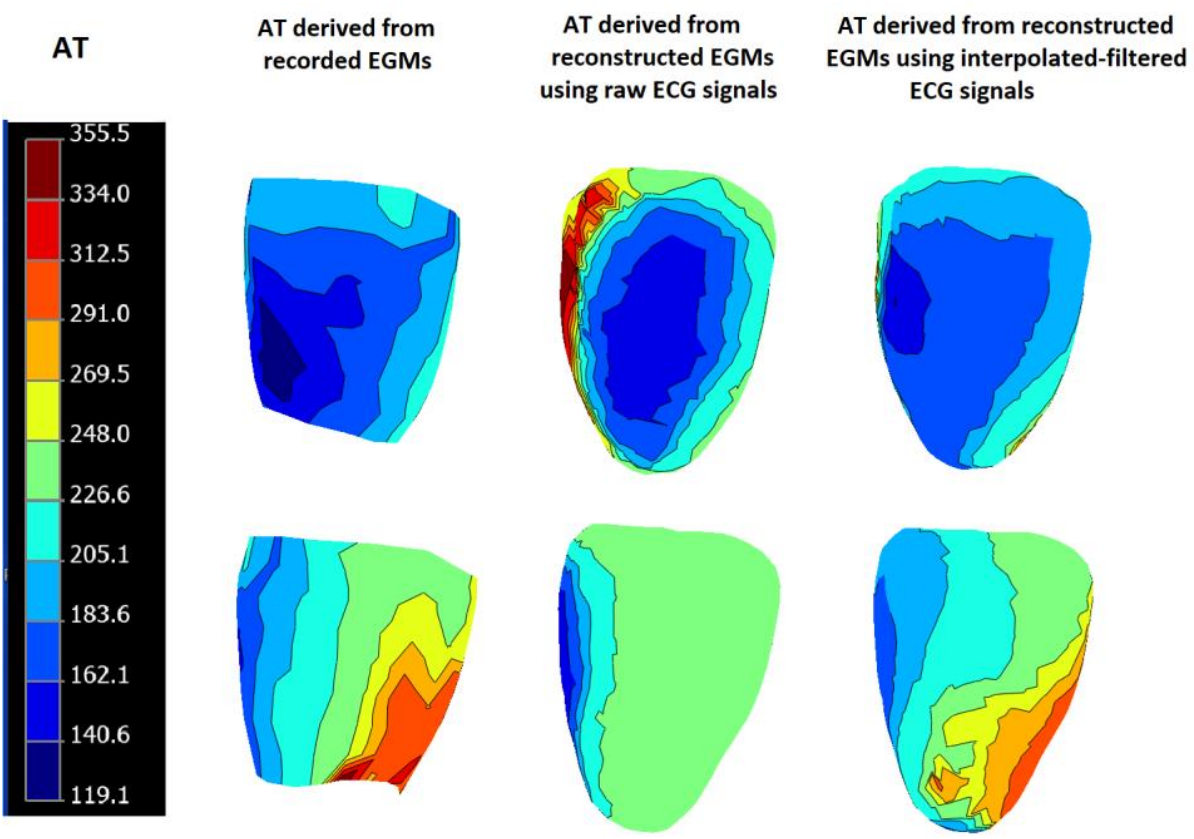

Fig. 7. Activation maps derived from recorded (left) and reconstructed electrograms using raw fullset (middle) and filtered LA-interpolated (right) torso signals for the fifth selected beat. [12].

Bear et al have shown in their work about the impact of filtering on inverse reconstruction of epicardial potentials that the removal of high frequency noise by applying signal averaging or low pass filtering improved the computation of activation times 
[9]. This suggests investigating the effect of signal averaging and low pass filtering at $40 \mathrm{~Hz}$ combined with interpolating low amplitude signals on the reconstructed activation maps. In addition, other methods of interpolation such as inverse forward interpolation method[12] should be investigated in the future work to compare that with Laplacian method used in this study.

\section{Conclusions}

Filtering ECG signals according to international and American standards improves electrogram reconstruction. In addition, removal of low amplitude signals and replacing them with interpolated signals combined with filtering according to the standard significantly improves reconstructed electrograms and derived activation maps. This suggests the need for adopting these two pre-processing steps for consistently and accurately reconstructing of epicardial potentials and derived activation maps.

Acknowledgments. This project is supported by the European Union's INTERREG VA Programme, managed by the Special EU Programmes Body (SEUPB). This work is also supported by the French National Research Agency (ANR-10-IAHU04LIRYC),

\section{References}

1. Cluitmans, M., Brooks, D.H., MacLeod, R., Dössel, O., Guillem, M.S., van Dam, P.M., Svehlikova, J., He, B., Sapp, J., Wang, L., Bear, L.: Validation and Opportunities of Electrocardiographic Imaging: From Technical Achievements to Clinical Applications. Front. Physiol. 9, 1305 (2018). doi:10.3389/fphys.2018.01305

2. Bear, L.R., Huntjens, P.R., Walton, R.D., Bernus, O., Coronel, R., Dubois, R.: Cardiac electrical dyssynchrony is accurately detected by noninvasive electrocardiographic imaging. Hear. Rhythm. 15, 1058-1069 (2018). doi:10.1016/j.hrthm.2018.02.024

3. Burton, B.M., Tate, J.D., Erem, B., Swenson, D.J., Wang, D.F., Steffen, M., Brooks, D.H., Van Dam, P.M., MacLeod, R.S.: A toolkit for forward/inverse problems in electrocardiography within the SCIRun problem solving environment. Proc. Annu. Int. Conf. IEEE Eng. Med. Biol. Soc. EMBS. 267270 (2011). doi:10.1109/IEMBS.2011.6090052

4. Oostendorp, T.F., van Oosterom, A., Huiskamp, G.: Interpolation on a triangulated 3D surface. J. Comput. Phys. 80, 331-343 (1989). doi:10.1016/0021-9991(89)90103-4

5. Rababah, A.S., Finlay, D.D., Guldenring, D., Bond, R., Mclaughlin, J.: An Adaptive Laplacian Based Interpolation Algorithm for Noise Reduction in Body Surface Potential Maps.

6. Briller, S., Mortara, D.: Diagnostic electrocardiographic devices. (2000) 
7. Luo, S., Johnston, P.: A review of electrocardiogram filtering. J. Electrocardiol. 43, 486-496 (2010). doi:10.1016/j.jelectrocard.2010.07.007

8. Kligfield, P., Gettes, L.: Recommendations for the Standardization and Interpretation of the Electrocardiogram. J. Am. Coll. Cardiol. 49, (2007). doi:10.1016/j.jacc.2007.01.024

9. Bear, L.R., Dogrusoz, Y.S., Svehlikova, J., Good, W., Dam, E. Van, Macleod, R., Abell, E., Walton, R., Coronel, R., Haissaguerre, M., Dubois, R., Bordeaux, U. De: Effects of ECG Signal Processing on the Inverse Problem of Electrocardiography. Comput. Cardiol. Conf. 45, (2018)

10. Duchateau, J., Potse, M., Dubois, R.: Spatially Coherent Activation Maps for Electrocardiographic Imaging. IEEE Trans. Biomed. Eng. 64, 1149-1156 (2017). doi:10.1109/TBME.2016.2593003

11. MacLeod, R.S., Johnson, C.R.: Map3d: Interactive Scientiic Visualization for Bioengineering Data.

12. Burnes, J.E., Kaelber, D.C., Taccardi, B., Lux, R.L., Ershler, P.R., Rudy, Y.: A field-compatible method for interpolating biopotentials. Ann. Biomed. Eng. 26, 37-47 (1998). doi:10.1114/1.49 